\title{
Further Investigation on Chaos of Real Digital Filters
}

\author{
B. W. K. LING, F. C. K. LUK and P. K. S. TAM \\ Department of Electronic and Information Engineering \\ The Hong Kong Polytechnic University \\ Hung Hom, Kowloon, Hong Kong \\ Hong Kong Special Administrative Region, China
}

\begin{abstract}
This letter displays, via the numerical simulation of a real digital filter, that a finitestate machine may behave in a near-chaotic way even when its corresponding infinitestate machine does not exhibit chaotic behavior.
\end{abstract}

Keywords: real digital filter; finite-state machine; chaotic behavior 


\section{Introduction}

Chaos in digital filters is a phenomenon of implementational and applicational importance [2-4]. A thorough understanding of chaos aids the implementation of a digital filter, which avoids undesirable chaotic behavior [4]. On the other hand, chaotic characteristics in digital filters can be utilized to build cost-effective and possibly high-security nonlinear digital encoders/decoders for communication purpose [3]. Therefore, an extensive exploration of this phenomenon in the real world is essential for the employment of these two practicalities [3,4]. There was an investigation on chaos in real (finite-state) second-order digital filters [2] where the question was raised, "In what sense can a real digital filter be considered to exhibit chaotic behavior?” It was then discovered that, ‘the asymptotic behavior of a realworld digital filter having a wordlength of only 16 bits is virtually indistinguishable from that of an infinite wordlength digital filter' [2]. Moreover, [1] shows that infinite wordlength digital filters can exhibit chaotic behaviors. As a result, it was concluded in [2] that a real digital filter with a 'sufficiently large' wordlength can also exhibit chaotic behavior. This paper is intended to further the investigation by raising another question, "Would a finite-state machine behave in a near-chaotic way even when its corresponding infinite-state machine does not exhibit chaotic behavior?”

\section{Behavioral Difference of Finite and Infinite Wordlength Digital filters}

To explore on the above question, a finite wordlength digital filter, where its corresponding infinite wordlength digital filter exhibits no chaotic behavior, is considered. Now, we need to decide on the possible wordlength of which this real digital filter might exhibit a near-chaotic pattern. Since it was quoted previously [2] that a digital filter with a wordlength of more than 16 bits exhibits a trajectory pattern 
that is 'virtually indistinguishable' from that of an infinite wordlength digital filter, a wordlength of more than 16 bits should exhibit no chaotic behavior. On the other hand, due to the number of states inferred by the number of bits of a digital filter, a 'sufficiently large’ wordlength needs to be chosen for visibility of chaotic pattern on trajectories [2]. Therefore, wordlengths of less than 16 bits are examined in the following simulation.

The real digital filter example of [2] was simulated as shown in Fig. 1, where the input-output characteristic of the staircase 2's complement $L$-bit quantizer was as shown in Fig. 2 [2]. Fig. 4 shows the corresponding trajectory of the real digital filter for 31 bits (>16 bits), where it shows a non-chaotic pattern: a trajectory is on a single ellipse [1]. Hence, the corresponding infinite wordlength digital filter is non-chaotic. However, the trajectories of filters with wordlength of 13 and 16 bits shown in Figs. 3 and 5, respectively, exhibit a near-fractal pattern and thus exhibits a near-chaotic behaviors [1].

\section{Conclusion}

From the trajectories simulated, a finite wordlength digital filter may behave in a near-chaotic way even when its corresponding infinite wordlength digital filter is nonchaotic. 


\section{Acknowledgement}

The work described in this paper was substantially supported by The Hong Kong Polytechnic University. 


\section{References}

[1] Chua, L. O. \& Lin, T. [1988], "Chaos in digital filters," IEEE Trans. Circuits Syst., vol. 35, pp. 648-658.

[2] Chua, L. O. \& Lin, T. [1991], "On chaos of digital filters in the real world," IEEE Trans. Circuits Syst., vol. 38, pp. 557-558.

[3] Frey, D. R. [1993], “Chaotic digital encoding: an approach to secure communication,” IEEE Trans. Circuits Syst. II, vol. 40, pp. 660-666.

[4] Dettmer R. [1993], “Chaos and Engineering,” IEE Review, vol. 39, p. 199-203. 


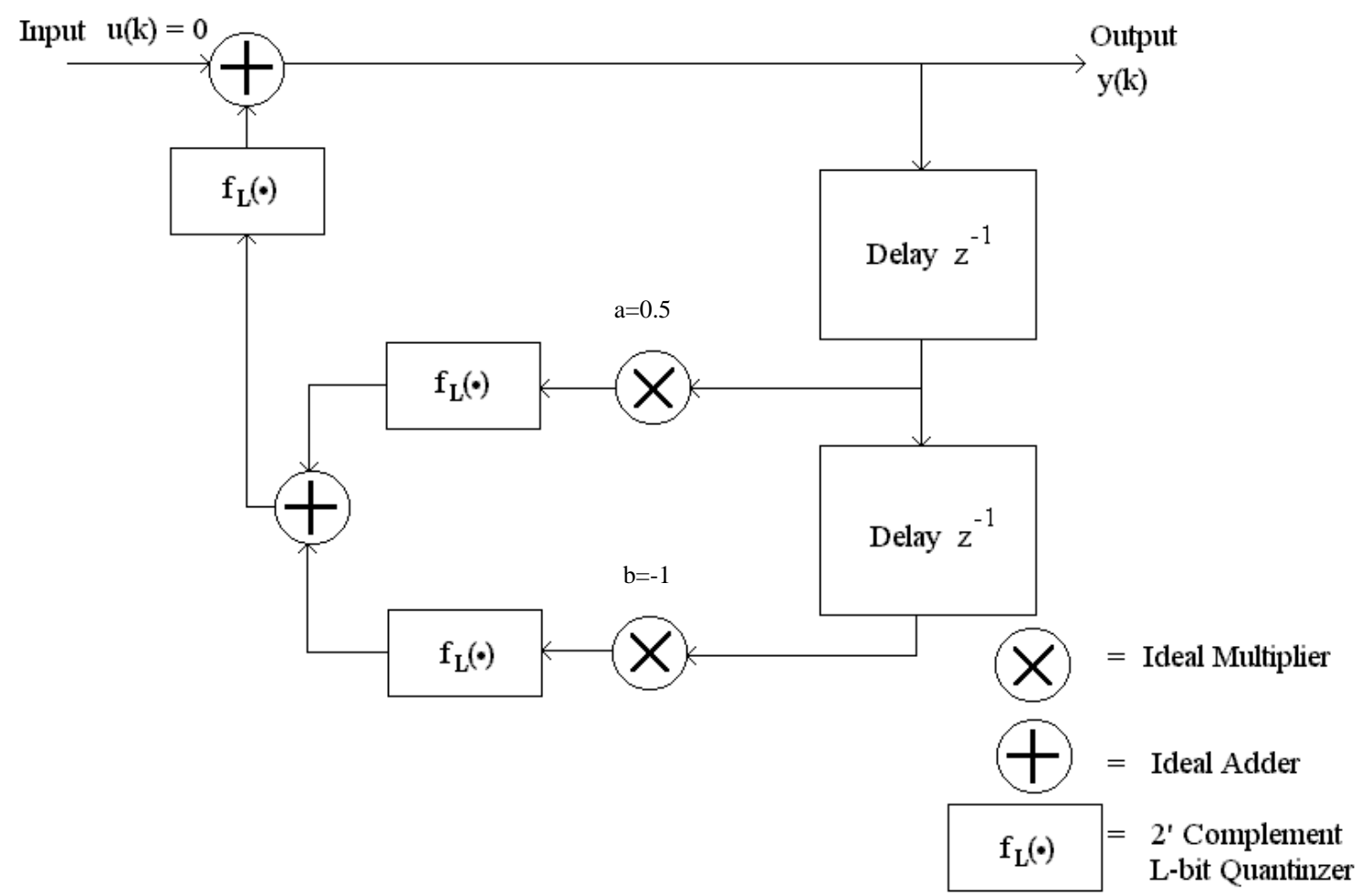

Fig. 1. A model of second-order digital filter with finite wordlength.

Authors: Wing-Kuen LING, Fiona Chi-Kwan LUK and Peter Kwong-Shun TAM 


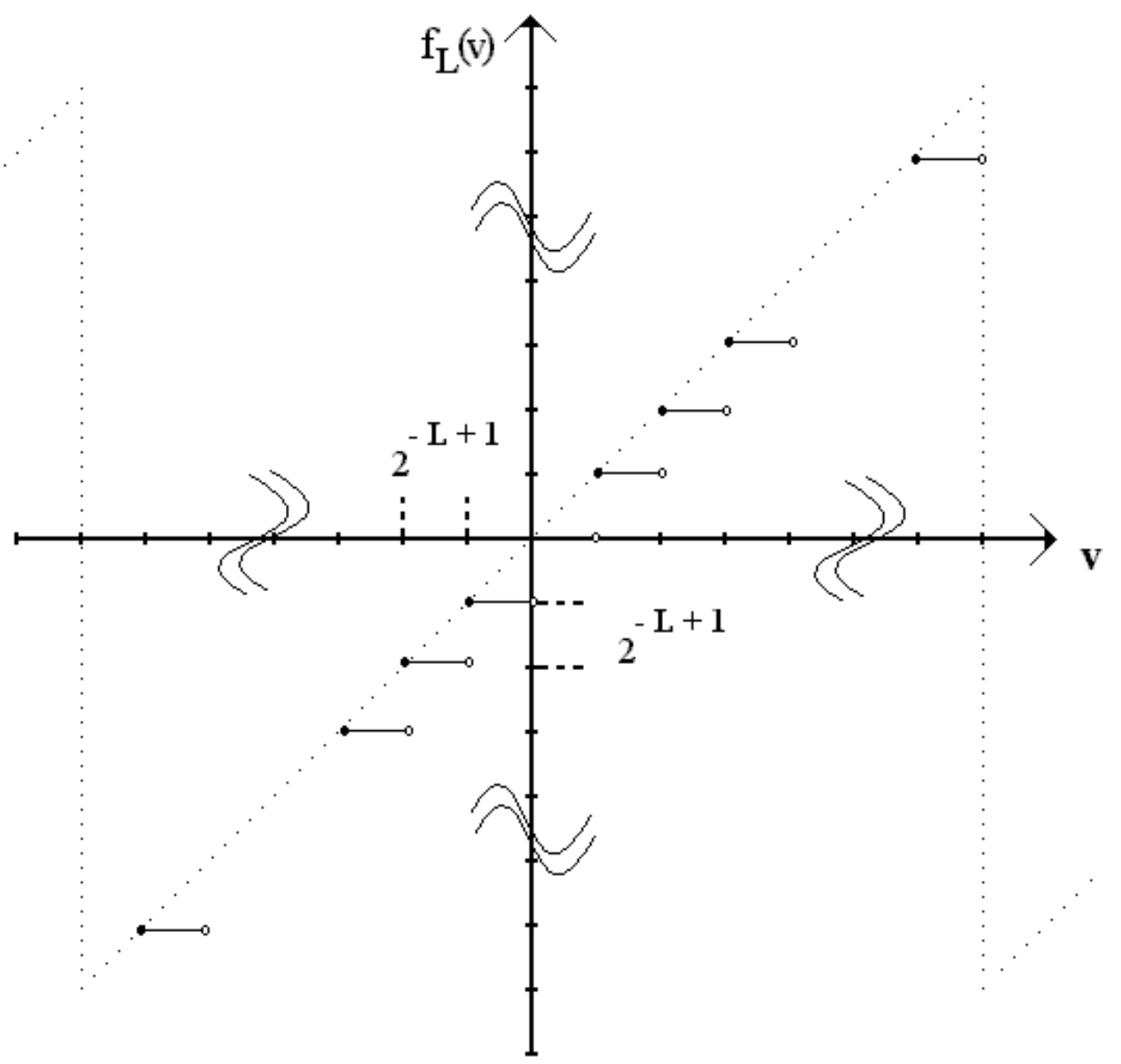

Fig. 2. The input-output characteristic of the 2's complement $L$-bit quantizer. 


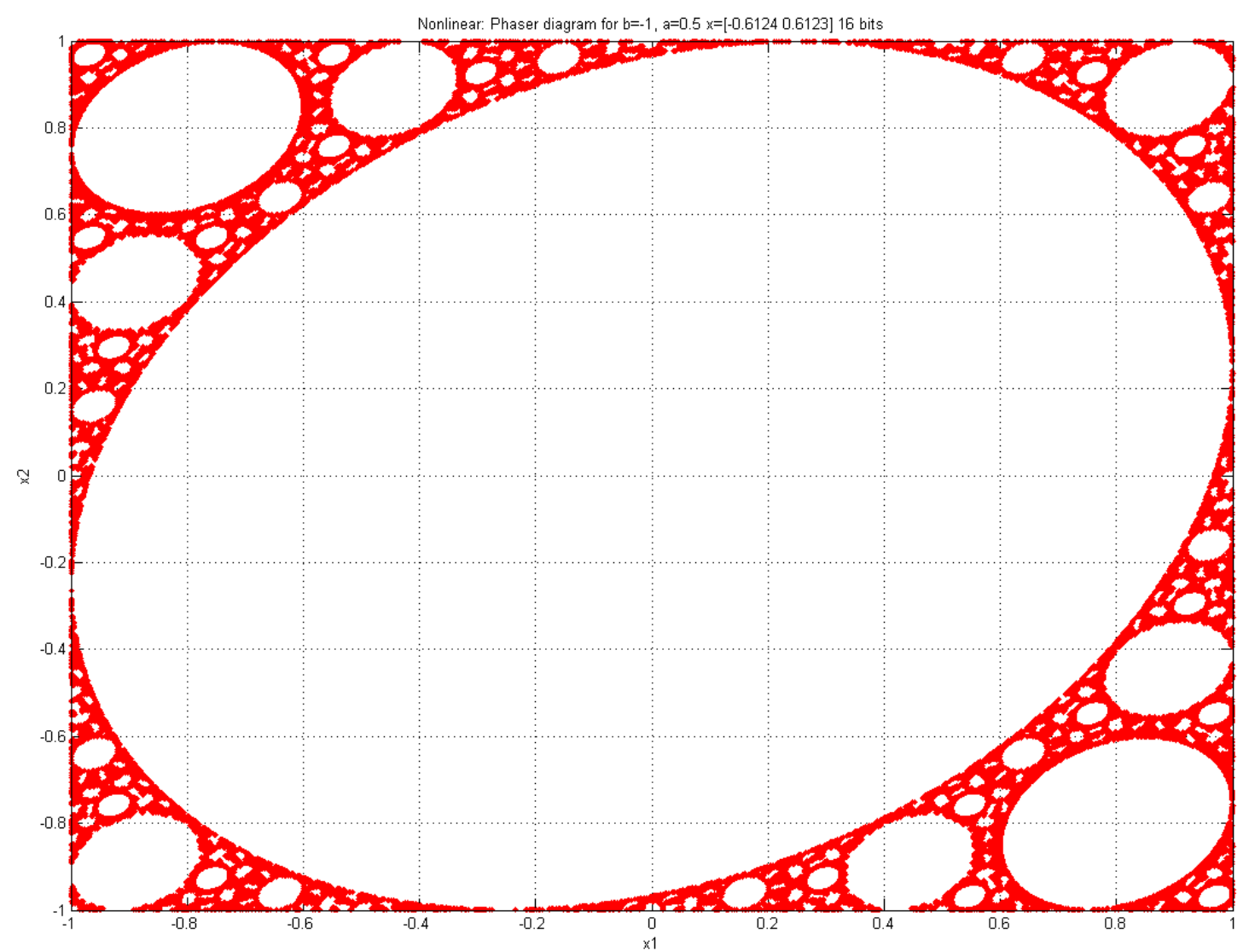

Fig. 3. A trajectory from 16-bit wordlength digital filter example.

Authors: Wing-Kuen LING, Fiona Chi-Kwan LUK and Peter Kwong-Shun TAM 


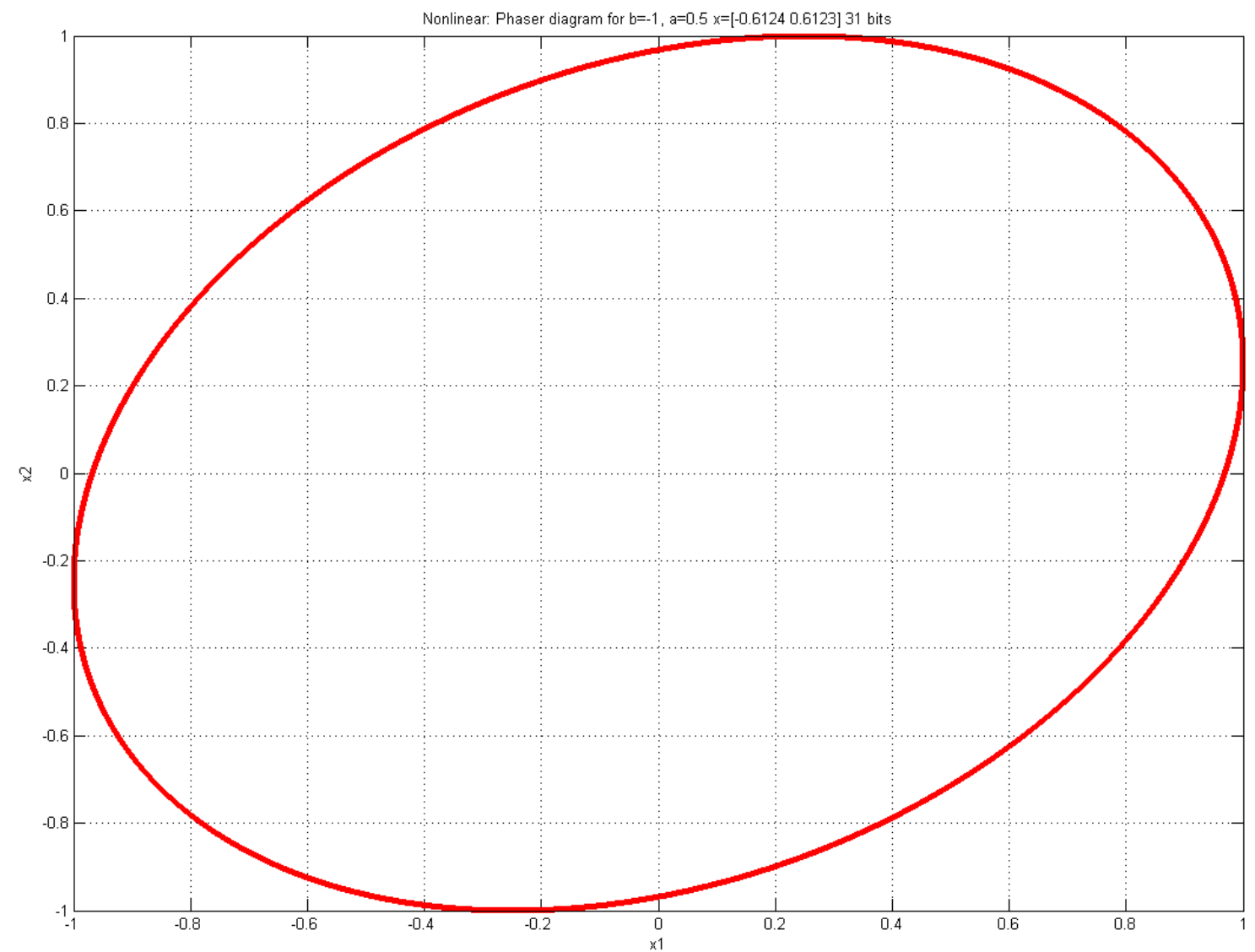

Fig. 4. A trajectory from 31-bit wordlength digital filter example.

Authors: Wing-Kuen LING, Fiona Chi-Kwan LUK and Peter Kwong-Shun TAM 


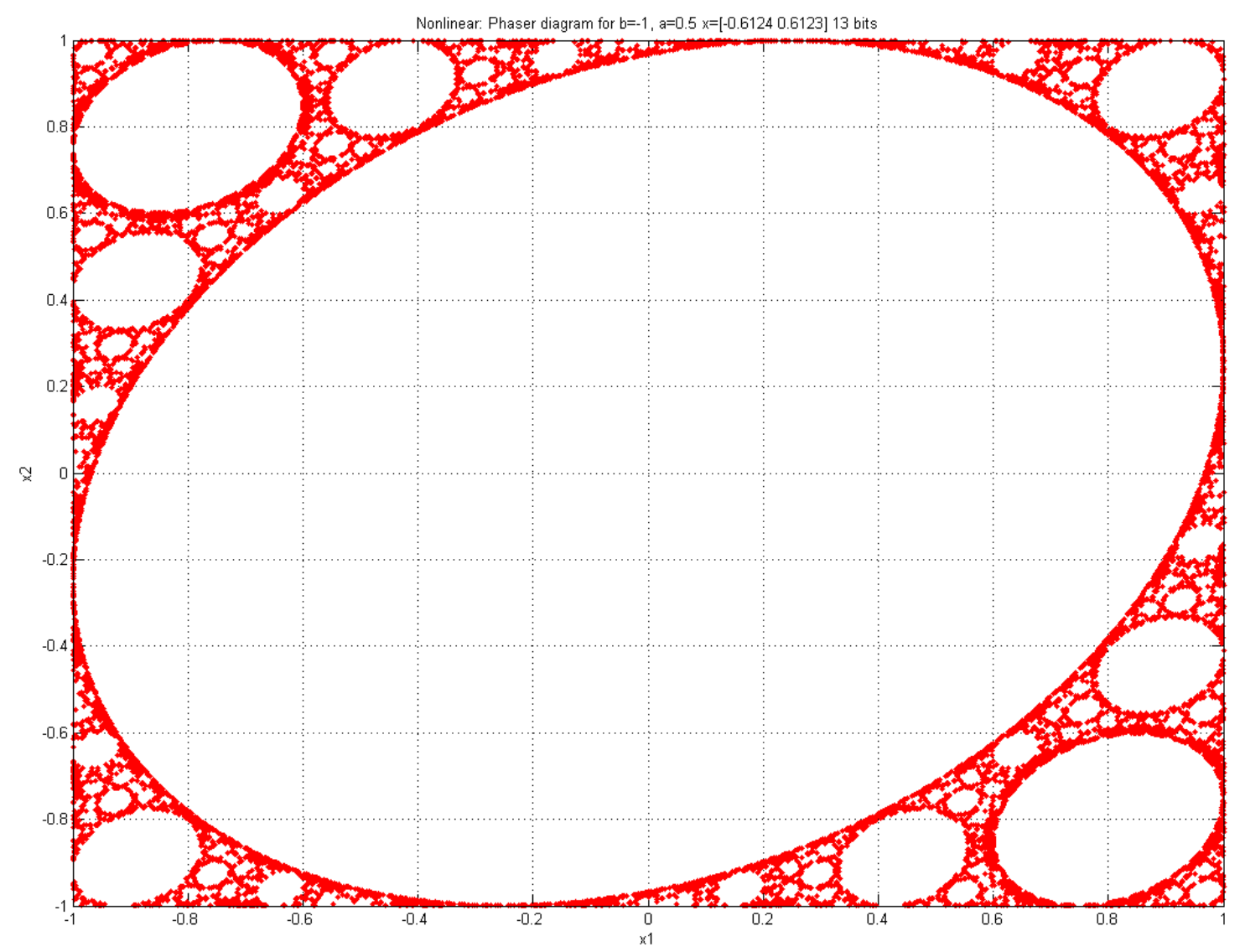

Fig. 5. A trajectory from 13-bit wordlength digital filter example.

Authors: Wing-Kuen LING, Fiona Chi-Kwan LUK and Peter Kwong-Shun TAM 